\title{
OBSERVATIONS OF INFRARED FINE STRUCTURE EMISSION
}

J.H. Lacy, F. Baas, S. Beck, and C.H. Townes University of California, Berkeley

A new spectrometer has been built for use in the $10 \mu \mathrm{m}$ atmospheric window which is substantially more sensitive than previous instruments for observations of $10 \mu \mathrm{m}$ emission and absorption features narrower than about $.25 \mathrm{~cm}^{-1}$. Measurements have been made of fine structure emission lines in planetary nebulae as well as in galactic and extragalactic $\mathrm{H}$ II regions. The spectrometer consists of a liquid Helium cooled grating with a resolution of $1-4 \mathrm{~cm}^{-1}$ and a liquid Nitrogen cooled Fabry-Perot spectrometer which is scanned across the grating bandpass, resulting in a resolution of $.1-.2 \mathrm{~cm}^{-1}$. The system NEP is about $4 \times 10^{-14} \mathrm{~W} / \sqrt{\mathrm{Hz}}$, resulting in a minimum detectable line flux of about $10^{-19} \mathrm{~W} / \mathrm{cm}^{2}$, with a 6" aperture on the Lick $3 \mathrm{~m}$ telescope.

We have undertaken a survey of the fine structure lines of Ne II, $S$ IV, and Ar III from planetary nebulae. These lines give a measure of the abundance of the ions which is only weakly dependent on temperature and is essentially unaffected by interstellar absorption. Since $\mathrm{Ne}$ II and S IV have no visible transitions, the fine structure radiation gives the only measure of their abundances. So far, 19 planetaries have been observed, most of which lie between $0^{\mathrm{h}}-16^{\mathrm{h}}$. At least one line was detected in 7 of these objects. The complete survey will include about 50 of the brightest planetaries. Other programs in progress include studies of the velocity fields in the Ne II line in M82 and the galactic center. The results for the galactic center are especially interesting, showing substantial variations in the strength and Doppler shift of the line on a scale of several arc seconds. This work was supported by NASA Grants Nos. NGR 05-003-452 and NGL 05-003272 .

RADIO SPECTROSCOPY OF PLANETARY NEBULAE

Eric J. Chaisson

Harvard-Smithsonian Center for Astrophysics

The $H 110 \alpha$ radio recombination line has been observed toward the planetary nebulae NGC 7027, IC 418, and NGC 6543 in order to ascertain the physical characteristics of the bulk nebular gas. The observations of NGC 7027 confirm the earlier findings of Chaisson and Malkan (Ap.J., $\underline{210}, 108,1976$ ) and Churchwel1, Terzian and Walmsley (A\&A, 48, 331, 1976) 